\title{
Contribution des Systèmes d'Information Géographique (SIG) à l'analyse de l'approvisionnement en Eau Potable dans la Commune de Kétou au Sud du Benin
}

\author{
Rébecca A. N. LAWANI ${ }^{*}$, Gildas BOKO², Bernadin ELEGBEDE MANOU², \\ Isaline WERTZ ${ }^{4}$, Maëlle VERCAUTEREN DRUBBEL ${ }^{4}$ et \\ Nelly AHOUANGNIVO KELOME ${ }^{1}$
}

\author{
${ }^{1}$ Unité de recherche en Géochimie des Systèmes Aquatiques, Département de Géologie, Faculté des Sciences \\ et Techniques, Université d'Abomey-Calavi, Cotonou, Bénin. \\ ${ }^{2}$ Département de Géographie et Aménagement du Territoire, Faculté des sciences humaines et sociales \\ (FASHS), Université d'Abomey-Calavi (UAC) \\ ${ }^{3}$ Institut National de l'Eau, Laboratoire des sciences et Techniques de l'Eau/Département du Génie de l'Eau et \\ de l'Assainissement, Université d'Abomey-Calavi, \\ ${ }^{4}$ Laboratoire d'Analyse Géospatiale (ANAGEO), Institut de Gestion de l'Environnement et d'Aménagement du \\ Territoire (IGEAT), Université Libre de Bruxelles, Belgique. \\ *Auteur correspondant ; E-mail : rebecca.lawany@yahoo.fr; Tel. +22996922841
}

\begin{tabular}{lll}
\hline Received: 27-09-2020 & Accepted: 22-01-2021 & Published: 28-02-2021 \\
\hline
\end{tabular}

\section{RESUME}

Cette étude aborde le problème de l'approvisionnement en eau potable dans la Commune de Kétou au Sud du Bénin. L'objectif poursuivi était d'évaluer la distribution spatiale des ouvrages d'approvisionnement en eau potable et la satisfaction des populations de la Commune de Kétou par rapport à la demande en eau potable. La distribution spatiale, l'accessibilité et la surexploitation des ouvrages d'approvisionnement en eau potable fonctionnels ont été évalués grâce à la cartographie thématique et l'analyse spatiale. Les résultats obtenus indiquent que les infrastructures sont inégalement réparties sur le territoire de la Commune. L'arrondissement d'Adakplamè est le moins desservi en ouvrages hydrauliques tandis que l'arrondissement de Kpankou présente le plus grand nombre d'ouvrages. Plus de $30 \%$ des ouvrages disponibles sont en panne, ce qui crée une surexploitation des points d'eau fonctionnels. L'accessibilité aux différents ouvrages est acceptable seulement dans les arrondissements au Sud de la Commune. Les ouvrages fonctionnels sont surchargés par rapport aux normes de desserte en fonction du type d'ouvrage. Au vu de ces constats, il s'avère nécessaire de prévoir la réalisation de nouveaux ouvrages et de procéder à la réparation de certaines infrastructures en panne afin de faire face aux demandes croissantes en eau potable dans la Commune.

(C) 2021 International Formulae Group. All rights reserved.

Mots clés : Distribution spatiale, ouvrages hydrauliques, inégale répartition, Kétou, département du plateau. 


\title{
Contribution of GIS in the diagnosis of the drinking water supply system in the town of Kétou in south Benin
}

\begin{abstract}
This study addresses the problem of drinking water supply in the Municipality of Kétou in southern Benin. The objective was to assess the spatial distribution of drinking water supply structures and the satisfaction of the populations of the Municipality of Kétou in relation to the demand for drinking water. The spatial distribution, accessibility and overload of the functional drinking water supply structures were assessed using an approach based on thematic mapping and spatial analysis. The results obtained indicate that the infrastructures are unevenly distributed over the territory of the Municipality of Kétou. The district of Adakplamè is the least concentrated in hydraulic works while the district of Kpankou presents the greatest number of works. Accessibility as a function of the time required to reach the various structures is acceptable only in the districts to the south of the Commune. Functional structures are overloaded compared to service standards depending on the type of structure. In view of these findings, it is necessary to plan for the construction of new structures and to repair certain broken down infrastructures in order to cope with the increasing demands for drinking water in the Municipality.
\end{abstract}

(C) 2021 International Formulae Group. All rights reserved.

Keywords: Spatial distribution, hydraulic works, unequal distribution, Kétou, plateau department.

\section{INTRODUCTION}

L'accès à une eau de boisson saine est une condition indispensable à la santé, un droit humain élémentaire et une composante clé des politiques efficaces de protection sanitaire. L'importance de l'eau, de l'assainissement et de l'hygiène pour la santé et le développement transparaît dans les conclusions d'une série de forums politiques internationaux, tels que la Conférence mondiale sur l'eau de Mar del Plata (Argentine), les objectifs du Millénaire pour le développement, adoptés par l'Assemblée générale des Nations Unies (ONU) en 2000 (Sokegbe et al., 2017). Dans le monde, 1,1 milliard de personnes sont dépourvues d'accès à des systèmes améliorés d'approvisionnement en eau de boisson. Cette faible disponibilité de l'eau potable dans les zones urbaines, périurbaines et rurales, contraint les populations à s'approvisionner en eau de puits et forages (Sokegbe et al., 2017).

Dans le cas du Bénin, pays de l'Afrique de l'Ouest en voie de développement, il existe encore des disparités importantes dans l'accès à l'eau potable. Seulement $43 \%$ de la population en milieu rural ont accès à l'eau contre $57 \%$ en milieu urbain. Ainsi, le déficit est de $57 \%$ et $43 \%$ respectivement en milieu rural et urbain (Kontinental Water Africa,
2018). Pourtant, le Bénin est un pays qui dispose sur toute sa superficie, de ressources en eau de surface et souterraines suffisantes pour assurer son développement socioéconomique, et cela pendant plusieurs années. Cette situation de faible taux de desserte en eau potable est due à l'insuffisance des points d'eau potable qui n'évolue pas en fonction de la croissance démographique. Elle résulte aussi de la vétusté et de la dégradation des installations existantes, ainsi que de la défaillance dans leur gestion (Laure, 2018). La Commune de Kétou, au Sud du Bénin ne fait pas exception et affiche un niveau de couverture en eau potable de 52,2\% en 2016 (Plan directeur, 2018).

Face à cette situation et en vertu du droit à l'eau potable de tous les citoyens où qu'ils se trouvent, le Gouvernement du Bénin a l'ambition d'assurer l'accès à l'eau potable à toute la population d'ici 2021 au lieu de 2030 ainsi que prévu par les objectifs de développement durable «ODD». C'est dans cette optique que la présente étude se veut être un outil d'aide à la décision pour les politiques et organes de prise de décision à travers l'utilisation des systèmes d'information géographiques (SIG). Le SIG joue un rôle 
primordial dans l'implantation, le suivi et la gestion des infrastructures d'AEP à travers le monde. Il permet également de structurer la donnée de façon logique pour répondre aux problématiques du métier de l'eau et de l'assainissement. Hormis sa fonction d'aide à la décision, c'est aussi un outil qui communique, en temps réel et à qui de droit, ce qui se passe sur le réseau (Gismartware, 2018). La mise en œuvre d'un SIG intégrant les informations sur les forages peut répondre aux besoins des gestionnaires, des investisseurs privés et des professionnels travaillant dans le domaine des ressources en eaux (KonanWaidhet et al., 2013).

Plusieurs études ont déjà permis de voir la nécessité de l'utilisation de cet outil pour une meilleure planification des ressources en eau en Afrique au Niger (Ibrahim, 2018) et en France (Nguyen, 2014). En République du Bénin (Houeze, 2017) en particulier dans la Commune de Zè (Hounguevou et al., 2017) et dans la Commune de Nikki (Abdoulaye, 2017), des travaux similaires ont été menés. Cependant, ces travaux font essentiellement une spatialisation des points d'eau disponibles et leur état de fonctionnement. D'autres études menées ont procédé par des enquêtes pour faire l'inventaire et une analyse des points d'eau disponibles dans les Communes (Michozounnou et al., 2015) mais n'ont pas réussi à aborder la problématique d'accès à l'eau en lien avec l'évolution démographique.

L'utilisation du SIG dans la présente étude permettra une meilleure visualisation de l'évolution démographique et des besoins en points d'eau à l'horizon 2023 dans la Commune de Kétou où aucune étude de ce type n'a encore été menée et facilitera la prise de décision des politiques et acteurs du secteur pour la réalisation des ouvrages d'approvisionnement en eau potable dans ladite Commune. Cette étude présente en un premier lieu la répartition des ouvrages d'AEP fonctionnels et non fonctionnels répandues sur l'étendue de la Commune, puis fait un diagnostic exhaustif de leur accessibilité et de leur surexploitation.

\section{MATERIEL ET METHODES}

\section{Cadre d'étude}

L'étude a été réalisée en République du Bénin dans la Commune de Kétou située à l'extrémité Nord du département du Plateau entre les latitudes $7^{\circ} 10$ et $7^{\circ} 41^{\prime} 17^{\prime \prime}$ Nord d'une part et les longitudes $2^{\circ} 24^{\prime} 24^{\prime \prime}$ et $2^{\circ} 47^{\prime} 40^{\prime \prime}$ d'autre part (IGN, 1963). Elle s'étend sur une superficie de $1775 \mathrm{~km}^{2}$ (INSAE, 2016), soit $1,55 \%$ du territoire national et $54,38 \%$ du département du Plateau. Cette Commune est limitée au Nord par la Commune de Savè, au Sud par la Commune de Pobè, à l'Ouest par les Communes de Ouinhi et de Zagnanado et à l'Est par la République Fédérale du Nigéria (Figure 1). La Commune est subdivisée en six arrondissements que sont Adakplamè, Idigny, Kétou, Kpankou, Odomèta, et Okpomèta. Ces arrondissements sont subdivisés en vingt-huit (28) villages et dix (10) quartiers de ville. Le climat qui règne dans la ville est de type subéquatorial à régime pluviométrique bimodal à deux nuances : grande saison de pluie (Mars à Juillet); petite saison de pluie (Septembre et Octobre); grande saison sèche (Novembre à Février); petite saison sèche (Août) (Adjovi et al., 2019).

\section{Données}

La méthodologie utilisée dans cette étude s'articule autour de trois (3) principales phases à savoir l'acquisition des données via différentes sources, le traitement de ces données et l'analyse des résultats qui en découlent. Une gamme variée de données a été utilisée. Il s'agit entre autres de données vectorielles (ouvrages d'approvisionnement en eau, routes, hydrographie, limites communales), des données raster (densités des populations obtenues via "Worldpop"), des données numériques et des données obtenues au travers de documents divers. Les données vectorielles ont été utiles pour la présentation de la zone d'étude dans son contexte. Les routes, l'hydrographie, limites communales ont été fournies par l'Institut Géographique National (IGN) du Bénin. Les données démographiques de la zone d'étude ont été acquises au niveau de l'Institut de la 
Statistique et de l'Analyse Economique (INSAE) du Bénin. Les données sur les ouvrages d'approvisionnement en eau potable (AEP) ont été collectées directement au niveau du service technique de la Mairie de Kétou, chargé des travaux d'Approvisionnement en Eau potable. Les données acquises ont subi un prétraitement avant leur utilisation dans le SIG. Les données sur les coordonnées géographiques, la nature des ouvrages d'AEP et leur état de fonctionnement ont été harmonisées dans le tableur Excel afin de faciliter leur projection et leur lecture. Après la définition du système de coordonnées de référence, ces données ont été importées et leur traitement a abouti à l'élaboration de diverses cartes thématiques et d'analyse réalisées dans les logiciels Quantum GIS (QGIS) et GRASS.

\section{Répartition, typologie et fonctionnalité des ouvrages d'approvisionnement en eau potable}

La répartition spatiale des infrastructures hydrauliques dans la Commune de Kétou en fonction du type d'ouvrage et en fonction de l'état de fonctionnement des ouvrages a été réalisée en plusieurs étapes représentées sur la Figure 2.

\section{Répartition spatiale des infrastructures par rapport à la densité de population et à la norme}

La distribution spatiale des ouvrages a été comparée en fonction de la densité de la population au sein de la Commune pour l'année 2018, afin de ressortir les arrondissements au sein de la Commune qui sont les plus dépourvus en ouvrages dans les zones de forte concentration en population. La densité utilisée ici est celle de Worldpop pour l'année 2018. Par ailleurs, les données de densités de populations obtenues ont été superposées aux couches "shapefiles" de la Commune, des routes, des cours d'eau et des ouvrages. Les informations ont été affichées et catégorisées. Dans cette étude, la densité de population (le plus souvent exprimé en individus par unité de surface) a été exprimée par le nombre d'habitants au kilomètre carré. Par ailleurs, pour apprécier l'arrondissement où les ouvrages sont concentrés, le nombre d'ouvrages a été apprécié par rapport à cette norme de desserte fixée par la stratégie nationale en AEP (DGEau, 2010) qui indique qu'il faut un point d'eau moderne pour 250 habitants et un AEV pour au moins 2000 habitants. Pour les AEV, on considère qu'une AEV a six (6) Bornes fontaines et une borne fontaine équivaut à deux (2) points d'eau.

\section{Élaboration des cartes d'accessibilité}

L'accessibilité a été évaluée en termes de distance à parcourir par la population et de temps nécessaire pour atteindre les différents ouvrages fonctionnels (Figure 3). Pour évaluer le temps à parcourir pour atteindre les ouvrages d'approvisionnement en eau potable fonctionnels, des isochrones ont été créés dans le logiciel GRASS par le module "v. isochrone" en utilisant l'hypothèse selon laquelle la vitesse de déplacement des populations à pied sur les routes est d'environ $6 \mathrm{~km} / \mathrm{h}$ contre $4 \mathrm{~km} / \mathrm{h}$ en dehors du réseau routier via les pistes et chemins non classifiés (Yemelong, 2018). Cette hypothèse a été formulée en tenant compte des connaissances sur la zone d'étude qui est une zone rurale fortement dominée par la pratique des activités champêtres et d'après Yemelong (2018). Les routes ne sont pas bitumées dans leur plus grande majorité. L'accessibilité en fonction du temps a été évaluée en $30 \mathrm{mn}, 1 \mathrm{~h}$, $2 \mathrm{~h}$ et $3 \mathrm{~h}$. Le réseau routier a été extrait d'Openstreetmap (OSM) par le site "Bbike' puis un nettoyage du réseau a été effectué par les modules "v. clean" et "v. generalize" de GRASS. Ensuite, ces couches ont été reprojetées du système de coordonnées de référence (SCR) WGS 84/EPSG 4326 au SCR WGS 84/EPSG 32631 UTM zone $31 \mathrm{~N}$. Les données utilisées pour réaliser la carte étant de type "qualitatives ordonnées", un symbole linéaire unique a été choisi. À l'issue de ces différents traitements, les couches constituant les données routières ont été superposées à celles des ouvrages d'eau potable.

Pour établir les cartes d'isodistances, la distance a été calculée à vol d'oiseau sur une surface d'une résolution de $100 \mathrm{~m}$. La distance constante entre différents contours est de 1000 $\mathrm{m}$. Les différentes cartes ont été réalisées en utilisant le logiciel GRASS puis le choix de la symbologie et la mise en page ont été réalisés 
dans le logiciel Quantum GIS(QGIS). La densité de la population utilisée en fond pour la carte montrant les villages desservis ou non desservis a été extraite du site Worldpop. Elle a permis de calculer la population par isochrone et par isodistance. Ces données ont subi un réajustement en tenant compte de la population réelle par arrondissement. Les modules utilisés sont le calculateur de raster "r.mapcalc" et le module 'v.rast.stats' qui permet d'obtenir des statistiques à partir d'une carte raster.

\section{Analyse de la surcharge des points d'eau}

Des zones tampons (buffer) ont été réalisées afin d'apprécier la distribution spatiale et de déterminer la zone de desserte des points d'eau (Ibrahim, 2018). Cette technique permet de visualiser les espaces où la couverture des infrastructures hydrauliques est très dense, donc l'accessibilité physique très bonne. Cette analyse est basée sur la norme nationale en vigueur pour la répartition des infrastructures hydrauliques. La norme de desserte fixée par la stratégie nationale en AEP (DGEau, 2010) est d'un point d'eau moderne (puits moderne ou forage équipé de pompe à motricité humaine) pour 250 habitants et d'un AEV pour au moins 2000 habitants. Tenant compte de cette norme, le nombre de population par pixel de la carte raster "Worldpop" à l'intérieur du buffer de $1000 \mathrm{~m}$ a été extrait puis comparé.

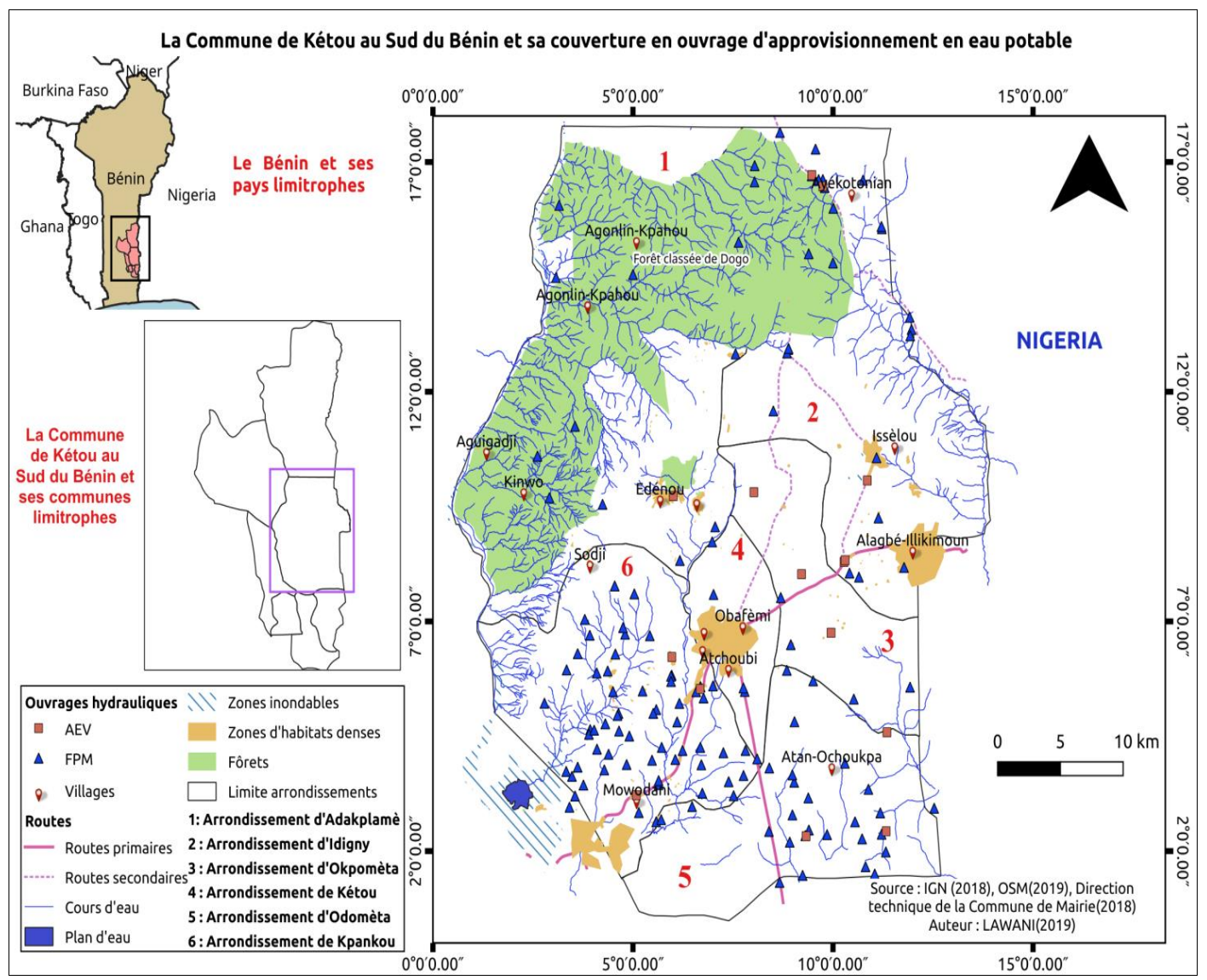

Figure 1: La Commune de Kétou et sa couverture en ouvrage d'approvisionnement en eau potable. 


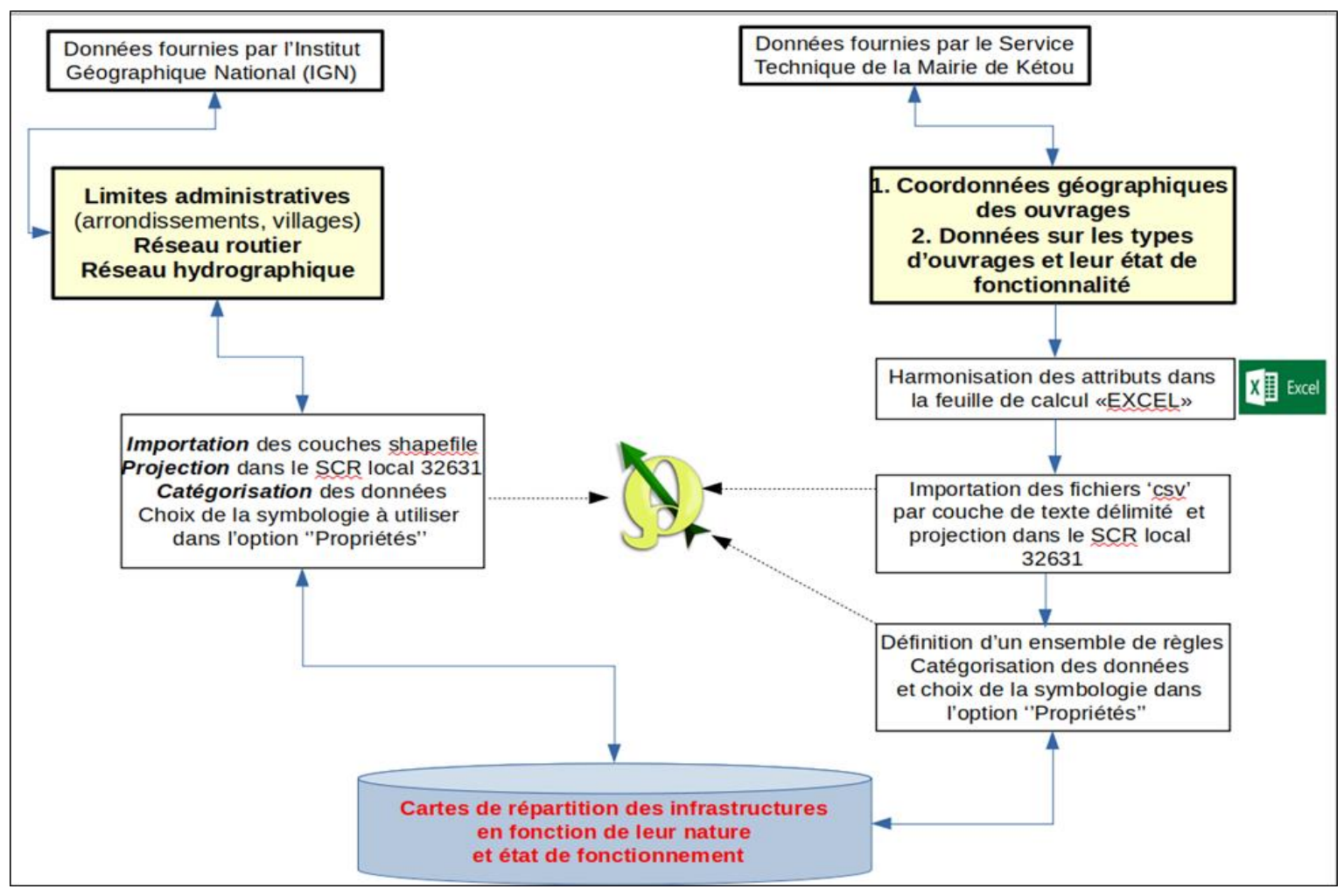

Figure 2 : Schéma opérationnel de réalisation des cartes inventaires.

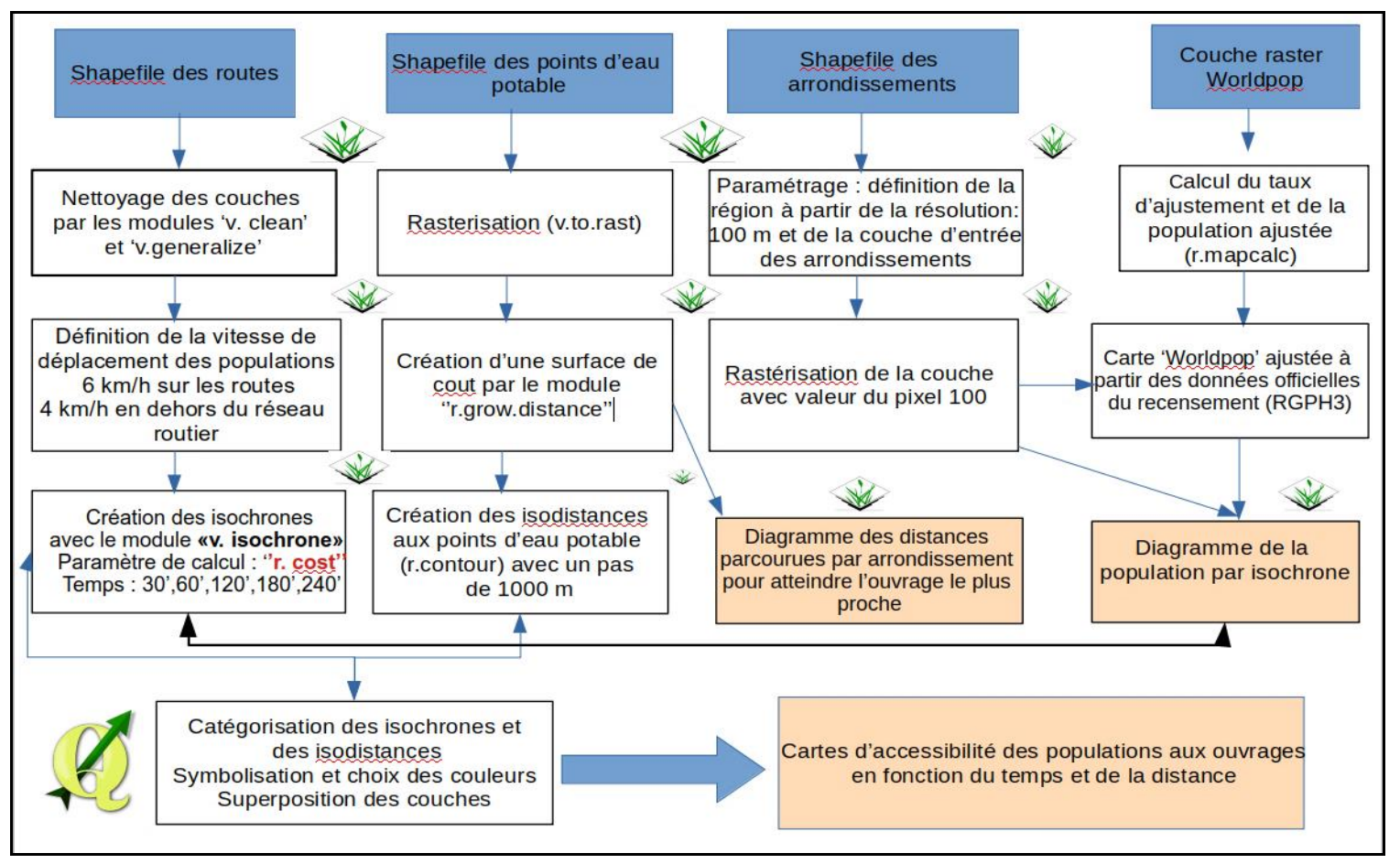

Figure 3: Schéma opérationnel de réalisation des cartes d'accessibilité aux ouvrages d'approvisionnement en eau potable. 


\section{RÉSULTATS}

\section{Typologie des infrastructures}

d'approvisionnement en eau potable dans la Commune de Kétou

$\mathrm{Au}$ total, cent cinquante points d'eau (150) ont été dénombrés dans la Commune d'étude (Figure 4). Il s'agit essentiellement de deux types d'ouvrages à savoir les forages à pompes motrices (FPM) qui sont au nombre de 135 et les adductions d'eau villageoises (AEV) qui sont au nombre de 15.

Répartition et état de fonctionnement ouvrages d'approvisionnement en eau potable

Parmi les points d'eau identifiés, certains nécessitent une intervention active de l'homme pour remplir leur rôle et d'autres non. Ils sont regroupés selon leur différent état de fonctionnalité dans le Tableau 1. De l'analyse du Tableau 2, il ressort que sur 132 forages à pompes motrices, 75 ne sont pas fonctionnels et 55 sont en bon état. Sur quinze (15) adductions d'eau villageoise, trois (3) sont non fonctionnels (en panne) et 12 sont fonctionnels (en bon état). En résumé, 41,66\% des ouvrages sont fonctionnels. $56,81 \%$ des forages à pompes motrices sont non fonctionnels contre seulement $20 \%$ des adductions d'eau villageoise qui ne fonctionnent pas. Les cartes des ouvrages fonctionnels et non fonctionnels ont été réalisées en fonction du type d'ouvrage (Figures 5, 6,7).

\section{Répartition des infrastructures suivant la densité de la population}

Les infrastructures d'approvisionnement en eau potable dans la Commune de Kétou sont mal réparties par rapport à la densité de la population (Figure 8). En effet, les arrondissements de Kétou et d'Idigny ont une forte densité de population mais c'est l'arrondissement de Kpankou qui dispose d'une forte concentration d'ouvrages hydrauliques (Figure 8). Quant aux arrondissements d'Adakplamè et d'Okpomèta, elles disposent d'une faible densité de population et d'une faible concentration en ouvrages hydrauliques. En termes de points d'eau, l'arrondissement le plus concentré est celui d'Idigny. Il contient le plus d'AEV et dessert donc une plus grande partie de la population.

Accessibilité des populations locales aux ouvrages d'approvisionnement en eau

L'accessibilité des populations locales aux ouvrages d'approvisionnement en eau a été déterminée dans un premier temps, en fonction de la distance qui sépare à vol d'oiseau le consommateur du point d'eau. Elle a été également déterminée en fonction du destemps, précisément la distribution de la population en fonction de la durée nécessaire pour aller à pied vers le point d'eau fonctionnel le plus proche. En fonction des facteurs et paramètres pris en compte, il ressort que $85 \%$ de la population totale (157352 habitants selon le quatrième recensement général de la population et de l'habitat) peut accéder en 30 minutes à pied à un ouvrage d'approvisionnement en eau potable fonctionnel (Figure 9). 11,78\% de la population met au moins une heure (60 minutes) pour avoir accès aux ouvrages d'eau fonctionnels et seulement $2,87 \%$ met au-delà d'une heure pour atteindre les ouvrages fonctionnels. La distance parcourue par les populations de l'arrondissement d'Adakplamè varie de 8000 jusqu'à $20000 \mathrm{~m}$ pour atteindre un point d'eau fonctionnel. Ces distances font ressortir une accessibilité inégalement répartie par arrondissement.

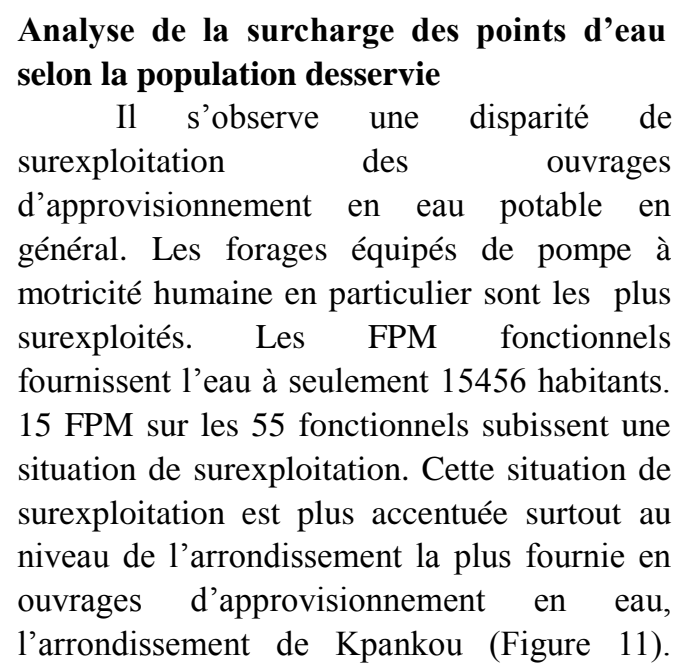




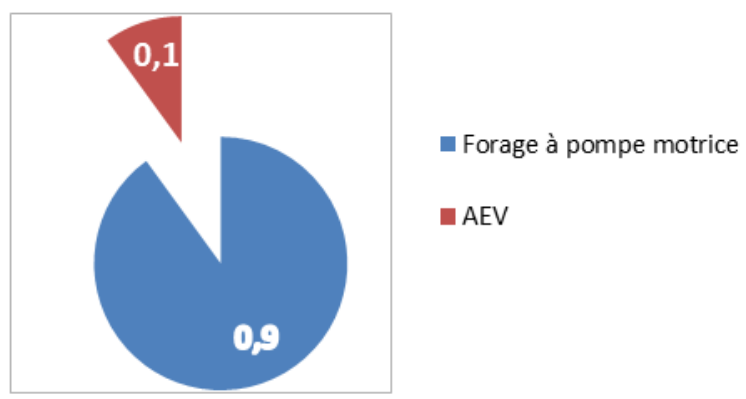

AEV : adduction d'eau villageoise

Figure 4: Types d'ouvrages dans la Commune de Kétou (Source : Données de la direction des services techniques de la mairie de Kétou, 2019).

Tableau 1: Nombre d'ouvrages fonctionnels et non fonctionnels.

\begin{tabular}{|c|c|c|c|c|c|}
\hline État & $\begin{array}{c}\text { Forage à pompe } \\
\text { motricité } \\
\text { humaine(FPM) }\end{array}$ & $\begin{array}{c}\text { Pourcentage } \\
\text { (FPM) }\end{array}$ & $\begin{array}{l}\text { Adduction d'Eau } \\
\text { Villageoise(AEV) }\end{array}$ & $\begin{array}{l}\text { Pourcentage } \\
\text { (AEV) }\end{array}$ & $\begin{array}{c}\text { Total }= \\
147\end{array}$ \\
\hline Fonctionnel & 55 & $41.6 \%$ & 12 & $80 \%$ & \\
\hline $\begin{array}{l}\text { Non } \\
\text { fonctionnel }\end{array}$ & 75 & $56.81 \%$ & 3 & $20 \%$ & \\
\hline Inconnu & 2 & $1 \%$ & 0 & 0 & \\
\hline Total & 132 & $99.41 \%$ & 15 & 100 & \\
\hline
\end{tabular}

Source : Données de la direction des services techniques de la mairie de Kétou, 2019.

Tableau 2 : Nombre de points d'eau par arrondissement.

\begin{tabular}{lccc}
\hline Arrondissements & Population en 2013 & Population totale en 2018 & $\begin{array}{c}\text { Nombre de points d'eau } \\
\text { en 2018 }\end{array}$ \\
\hline Odometa & 14802 & 17088 & 46 \\
\hline Kpankou & 27078 & 31260 & 74 \\
\hline Idigny & 46444 & 53617 & 110 \\
\hline Adakplame & 20218 & 23341 & 21 \\
\hline Okpometa & 9184 & 10602 & 53 \\
\hline Ketou & 39626 & 45746 & 310 \\
\hline Total & 157352 & 181654 & 6 \\
\hline
\end{tabular}




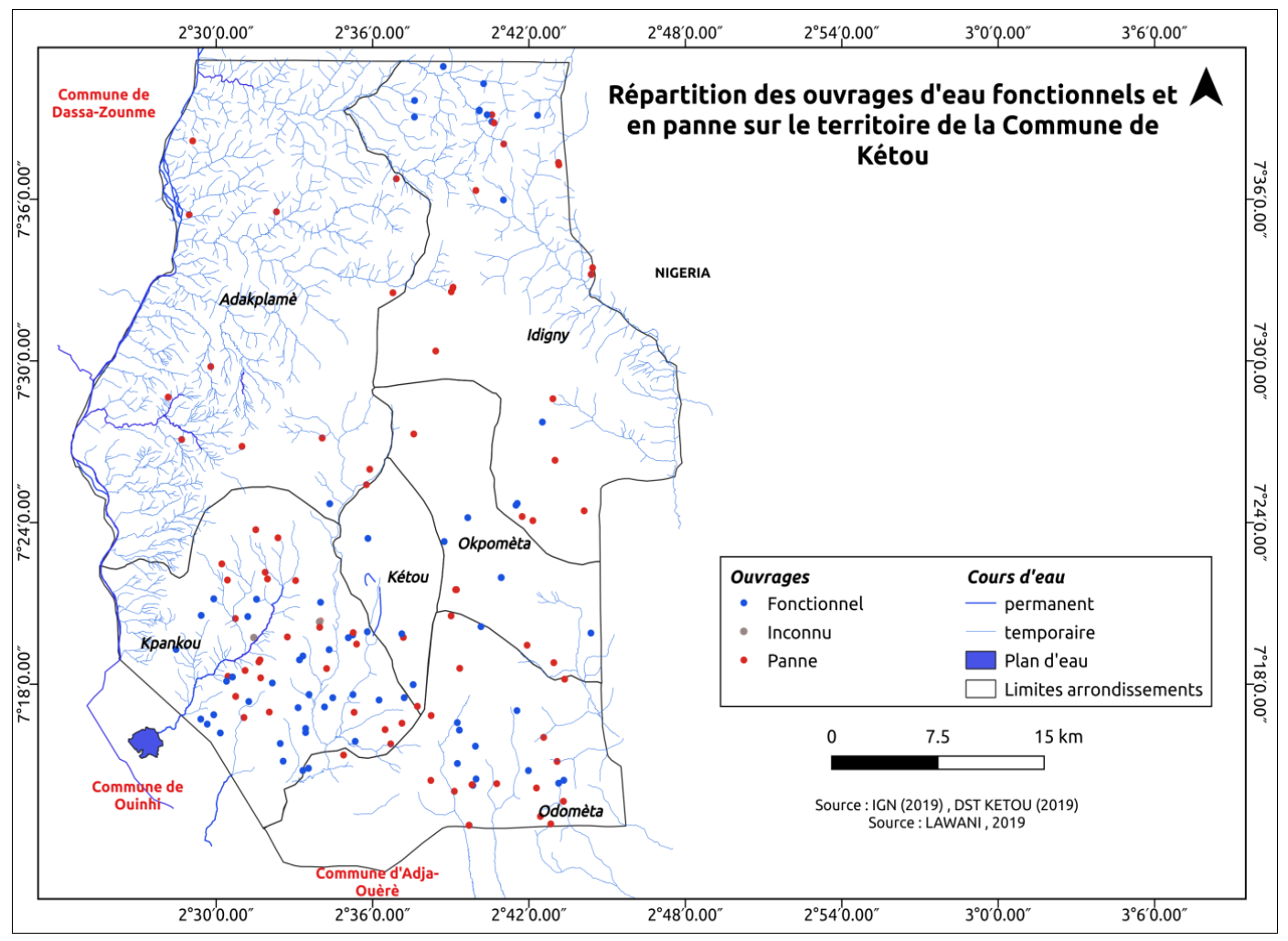

Figure 5 : Répartition spatiale générale des infrastructures hydrauliques fonctionnelles et non fonctionnelles dans la Commune de Kétou.

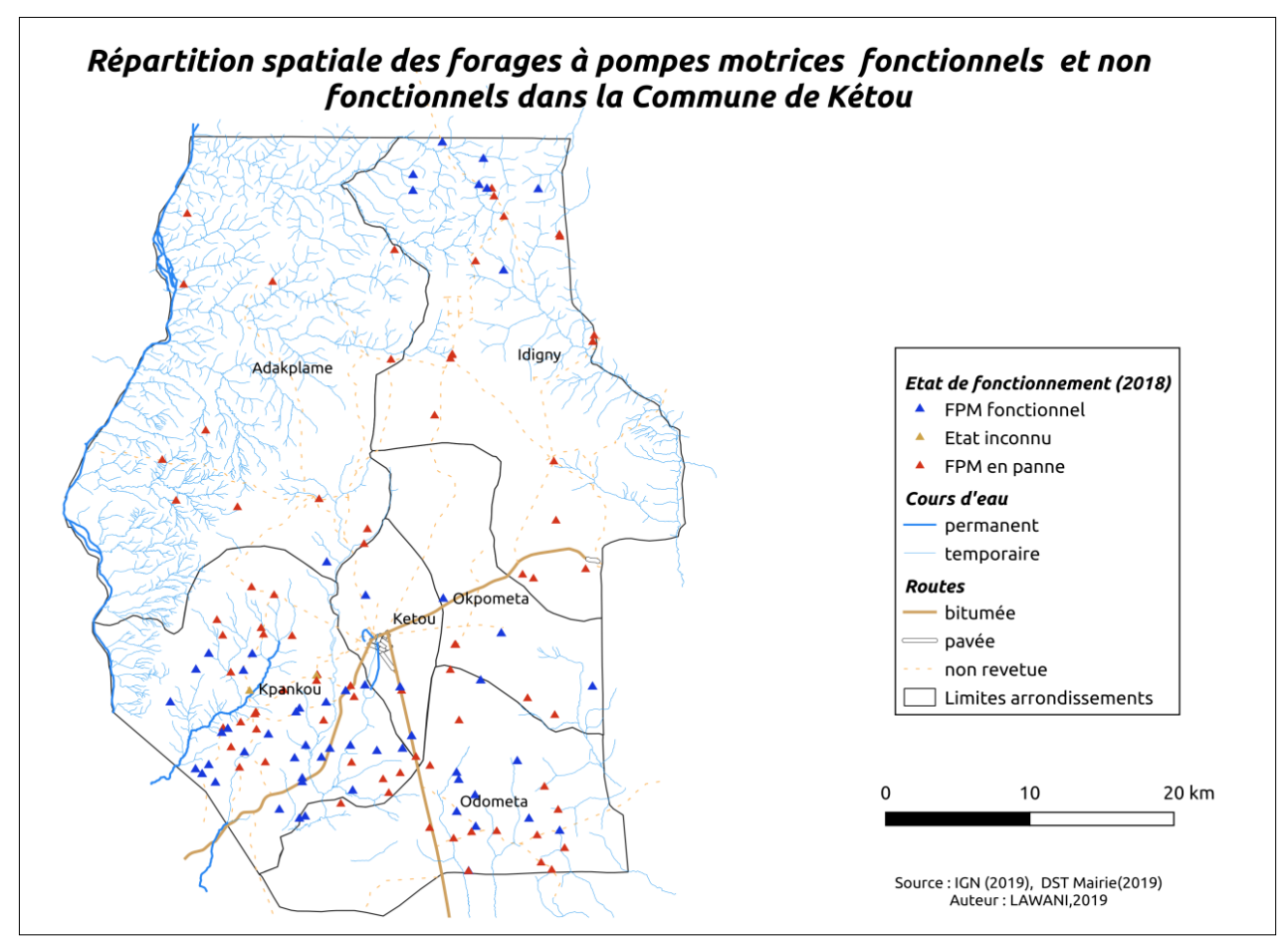

Figure 6: Répartition des forages à pompes motrices fonctionnels et en panne dans la Commune de Kétou. 


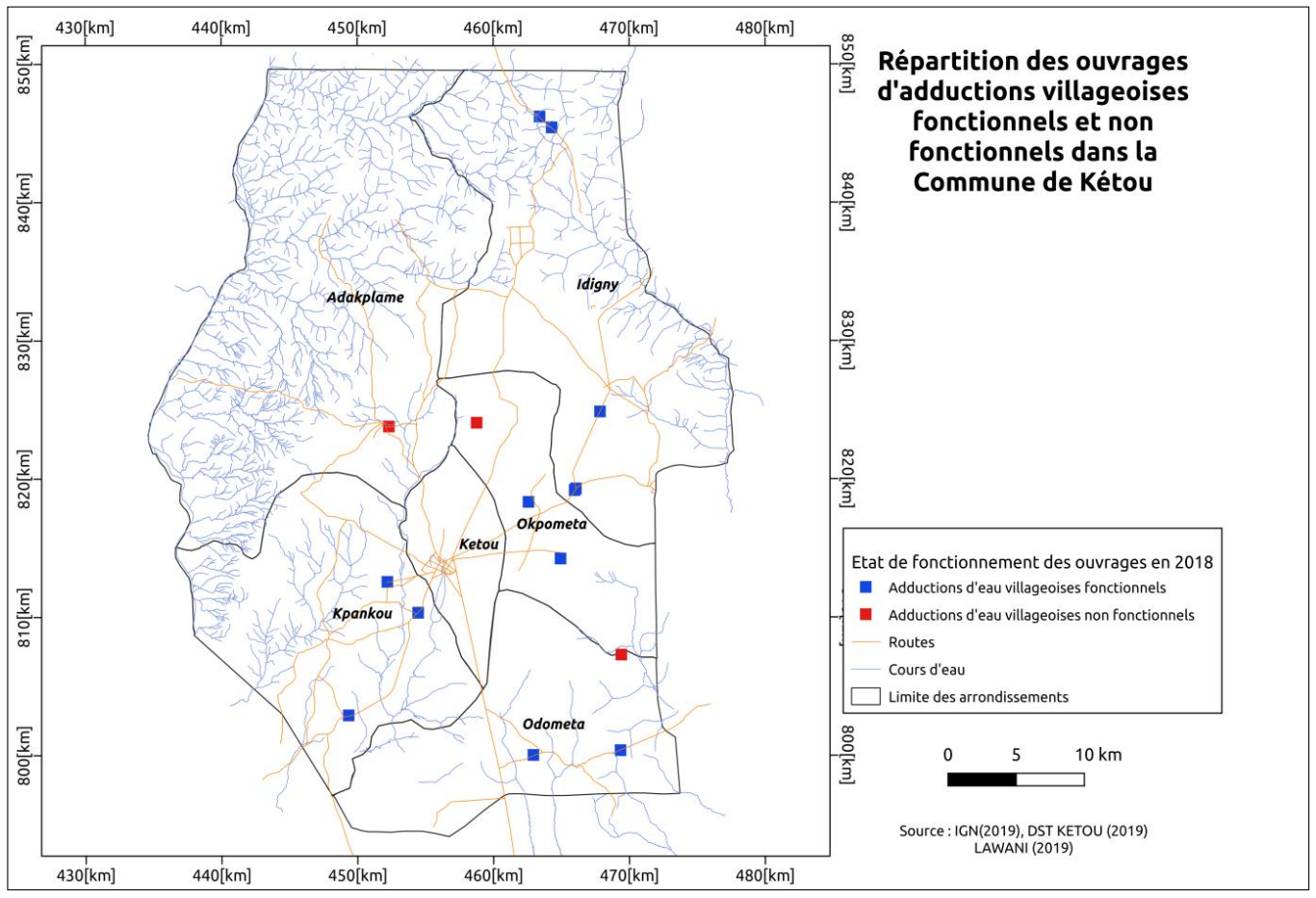

Figure 7: Répartition des adductions d'eau villageoise fonctionnelles et en panne dans la Commune.

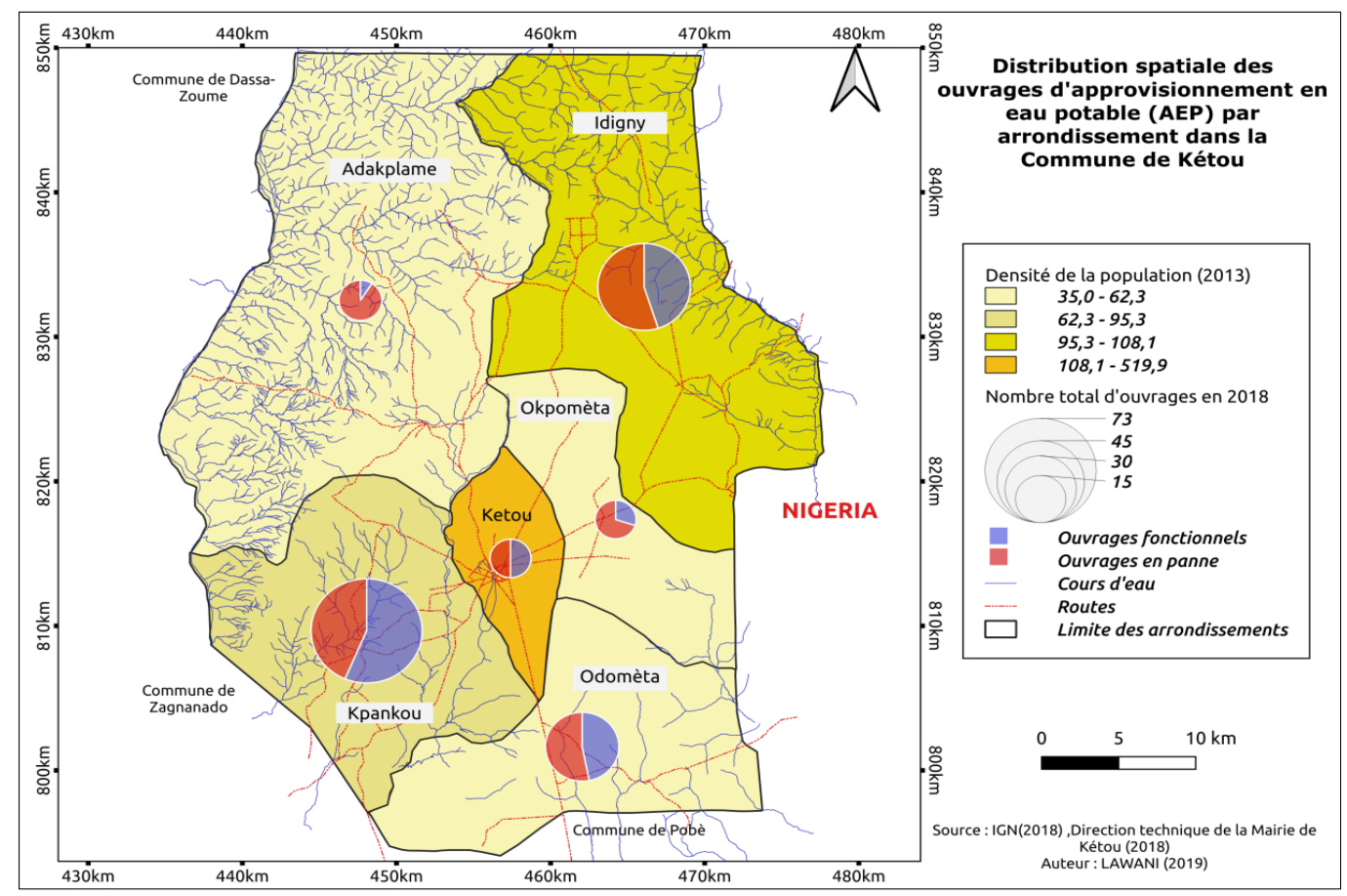

Figure 8: Distribution spatiale des ouvrages et densité de la population estimée en 2018 selon les données Worldpop. 


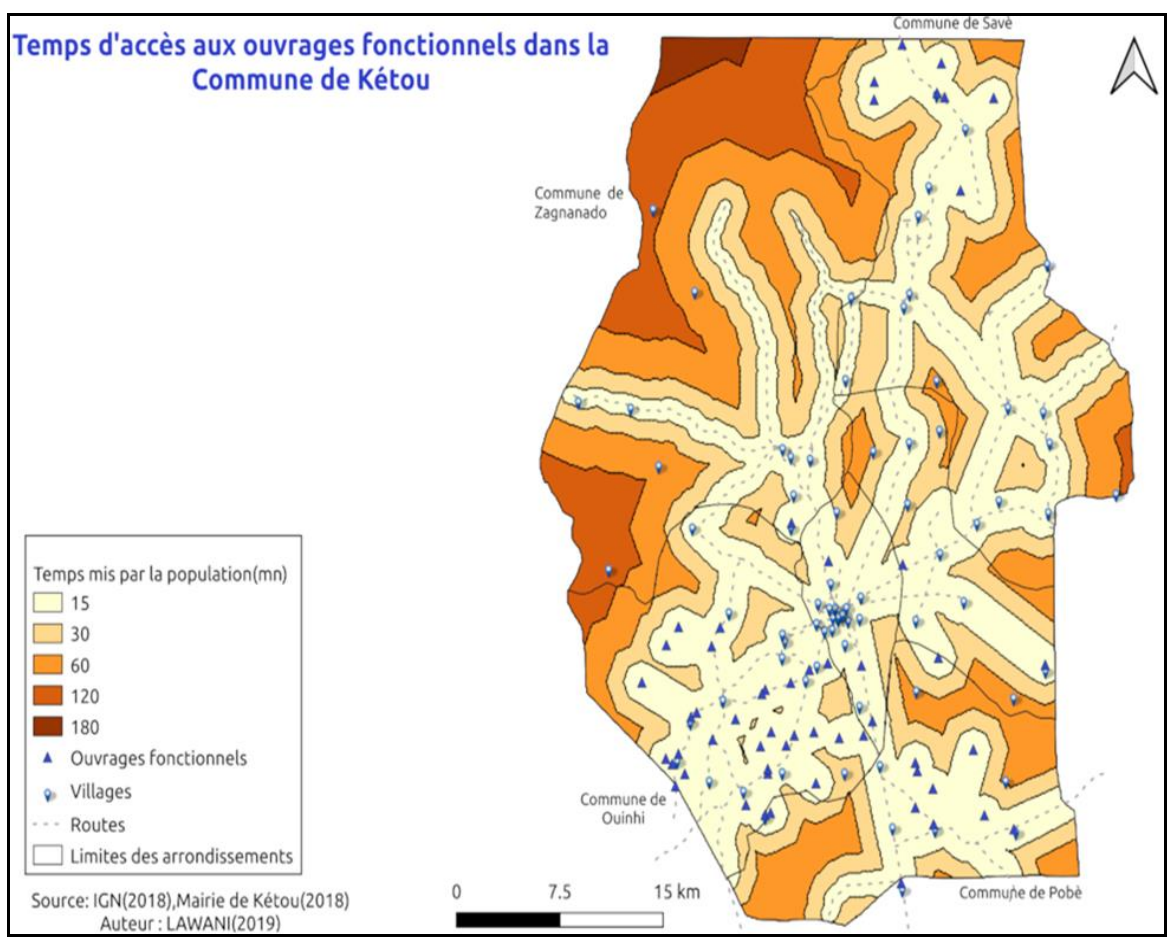

Figure 9: Temps nécessaire pour atteindre à pied les points d'eau potables fonctionnels dans la Commune de Ketou (vitesse de déplacement $4 \mathrm{~km} / \mathrm{h}$ ).

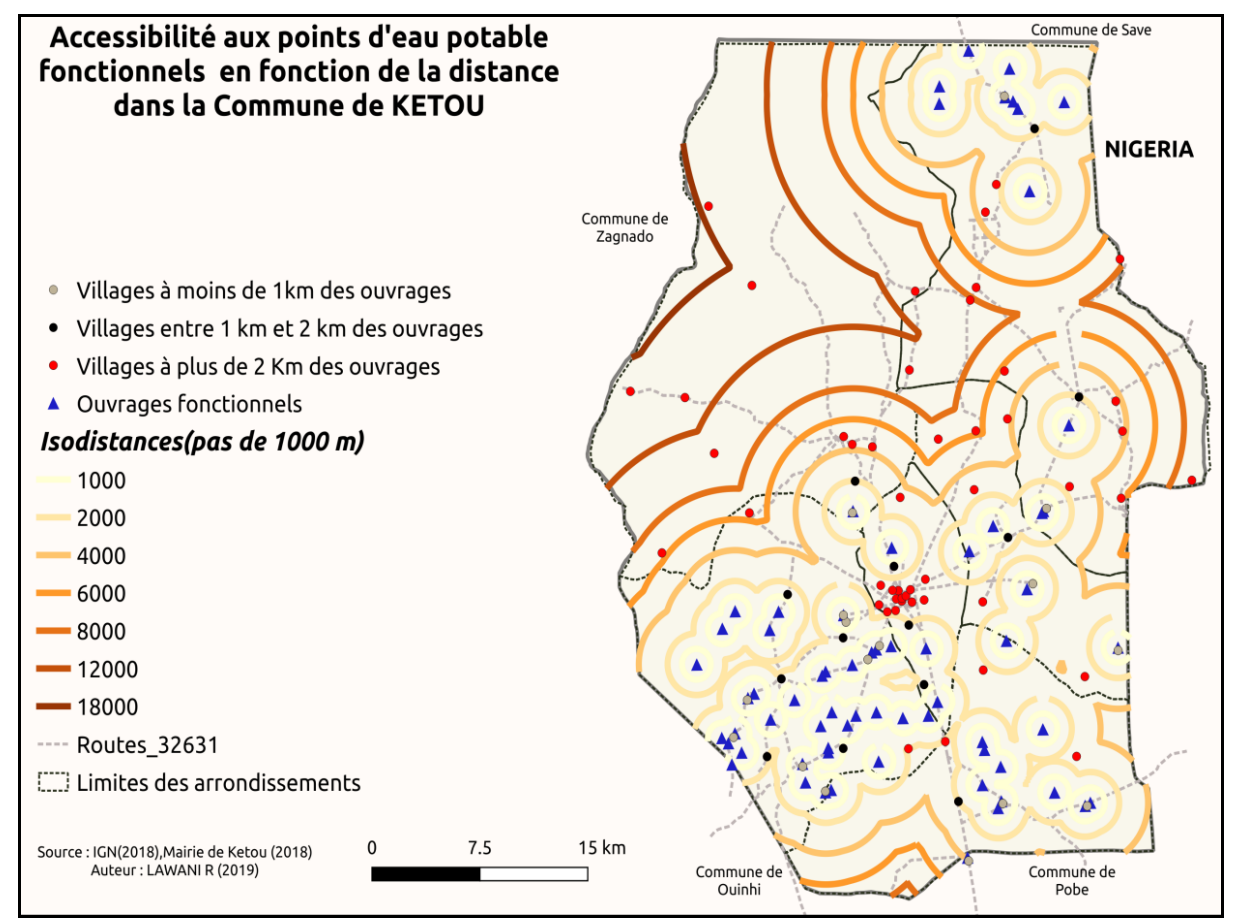

Figure 10: Distance $(\mathrm{m})$ à parcourir à partir de chaque arrondissement pour atteindre les points d'eau fonctionnels les plus proches dans la Commune de Kétou (vitesse de déplacement $=>6 \mathrm{~km} / \mathrm{h}$ ). 


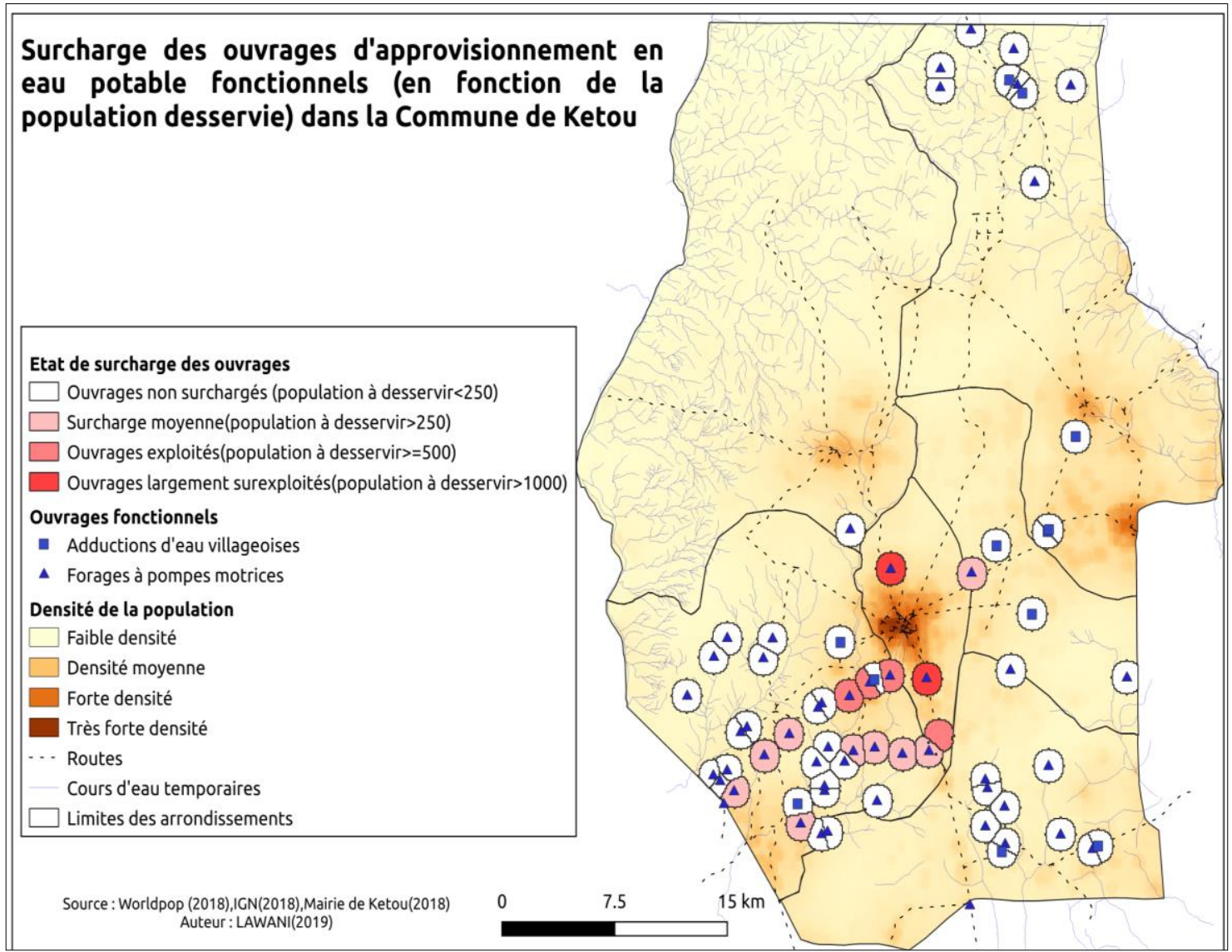

Figure 11: État de surcharge des ouvrages d'approvisionnement en eau potable fonctionnels en fonction de la population desservie.

\section{DISCUSSION}

La situation de l'accès à l'eau potable est encore loin d'être satisfaisante dans la Commune de Kétou. Les ouvrages d'approvisionnement en eau potable utilisés dans la Commune sont principalement les adductions d'eau villageoises, les forages équipés de pompes à motricité humaine et les postes d'eau autonomes privés. L'adduction d'eau villageoise (AEV) est généralement constituée d'un forage équipé d'un système de pompage motorisé relié à un réservoir de stockage et à un réseau de distribution d'eau. L'eau est distribuée par des canalisations enterrées au moyen de bornes fontaines et de branchements particuliers. Les AEV sont caractérisées par une distance plus ou moins grande entre le lieu de captage et le lieu de distribution de l'eau. Elles permettent la suppression du pompage manuel, réduisent l'attente aux points de distribution de l'eau et rapprochent ces points de distribution des populations. Les adductions d'eau villageoises sont une bonne solution pour l'alimentation en eau potable des villages totalisant plus de 2000 habitants ou pour des groupes de villages et localités proches les uns des autres (Lawani, 2017). Les forages équipés d'une pompe à motricité humaine quant à eux, sont utilisés lorsque la population à desservir atteint 250 habitants. Dans la présente étude, les Postes d'Eau Autonomes (PEA) n'ont pas été pris en compte car appartenant à des particuliers. Ce sont des PEA privés à partir 
desquels l'eau est vendue aux populations (Kelome et al., 2014). La qualité de l'eau n'est pas toujours adéquate (Kelome et al., 2014). Les types de points d'eau identifiés sont des ouvrages conventionnels, utilisés pour les usages domestiques (boisson, cuisine, lessive).

Les résultats de la présente étude indiquent qu'en 2018, il subsiste encore de grandes disparités entre les différents arrondissements de la Commune. La répartition des ouvrages hydrauliques dans la Commune est inégale et ne tient pas compte de la densité de la population. Il n'est pas rare de noter la concentration de plusieurs infrastructures d'approvisionnement en eau potable au même endroit comme dans l'arrondissement de Kpankou car les populations à la base ne sont pas souvent associées au choix du point d'implantation des ouvrages d'approvisionnement en eau. Il en résulte parfois des situations de pénurie d'eau dans d'autres localités et l'orientation des populations vers les sources de qualité douteuse ou non aménagées pour gagner du temps. Ces sources sont souvent à l'origine de maladies hydriques telles que le choléra, la fièvre typhoïde et la diarrhée comme l'ont démontré de nombreuses études (Gnazou et al., 2015 ; Sokegbe et al., 2017). Le déficit d'infrastructures hydrauliques favorise aussi d'après Gnazou et al. (2015), la précarité des conditions d'apprentissage des élèves.

Par ailleurs, l'arrondissement d'Adakplamè est la moins concentrée en ouvrages d'approvisionnement en eau potable. Les facteurs qui pourraient expliquer cette situation sont la présence de nombreux cours d'eaux temporaires qui servent à la population comme source de boisson et la présence de forets sacrées. L'hydrogéologie pourrait également être un facteur primordial. Cette inégale répartition des ouvrages d'approvisionnement en eau potable a été également relevée dans d'autres Communes au Bénin, au Togo et au Cameroun par Hounguevou (2014), Abdoulaye (2017),
Takem (2017), Sokegbe et al. (2017) et Yemelong (2018).

L'inégale répartition des ouvrages est à la base de la surcharge constatée au niveau de certains ouvrages fonctionnels. Cette situation est surtout observée au niveau de l'arrondissement de Kpankou et peut s'expliquer par la densité forte de la population au sein de cet arrondissement et le nombre élevé d'ouvrages en panne dans ledit arrondissement. Dans la Commune, les forages équipés de pompes motrices sont surexploités. Cette situation s'explique surtout par le taux de panne des FPM de plus de $57 \%$. La surexploitation des ouvrages d'approvisionnement en eau potable a déjà été démontrée par d'autres auteurs dans plusieurs Communes du BENIN à savoir la Commune de Kandi (Assouma et al., 2013) et dans les arrondissements de Biro et Sèrékalé dans la Commune de Nikki (Abdoulaye, 2017). La surcharge des infrastructures hydrauliques crée souvent des files d'attente lors de l'approvisionnement et des pertes de temps pour les populations.

L'accessibilité à l'eau la plus faible a été enregistrée dans l'Arrondissement d'Adakplamè. Les populations de cet arrondissement notamment les femmes devront parcourir de longues distances à la recherche d'eau de boisson avec tous les risques qui y sont liés: le risque sanitaire compte tenu de la charge supportée, le risque d'agression au cours du trajet, et les faibles revenues de la gente féminine rurale. Ces risques sont remarqués en Asie, en Afrique et en Amérique latine où elles sont des millions à parcourir quotidiennement de longues distances pour prélever l'eau de puits et l'eau des fleuves éloignés, sacrifiant ainsi l'éducation des filles à la nécessité vitale de l'approvisionnement en eau (CRS, 2015).

Seule la construction de nouveaux points d'eau et l'extension du réseau de la SONEB permettront de corriger le déficit actuel au niveau de cet arrondissement et la situation économique des femmes, qui sont les 
premières impactées par ce déficit. Un effort devrait se faire pour associer aussi la population au choix des sites d'implantation et au choix de la forme desdits ouvrages comme le soutient Hounguevou (2014). Cela éviterait certainement les problèmes de grandes distances et favoriserait l'accès facile à une eau de bonne qualité.

Il est nécessaire de souligner que l'Arrondissement d'Adakplamè se trouve dans la zone dite hydrogéologiquement difficile. C'est pourquoi l'implantation des futurs ouvrages doit faire appel aux nouvelles méthodes et technologies géophysiques afin d'éviter les risques de réalisation de forages négatifs. Aussi, au regard de la densité du réseau hydrographique fluviale dans les arrondissements d'Adakplamè et d'Idigny, les agents du service technique de la mairie doivent sensibiliser la population aux modes simples de désinfection et de traitement des eaux à travers leur filtration ou leur ébullition. Les produits tels que "Aquatabs" pourraient être également conseillés après que l'eau ait été correctement décantée.

\section{Conclusion}

Afin d'atteindre l'objectif de développement durable (ODD) n ${ }^{\circ} 6$ d'ici 2030, les SIG semblent être l'outil le plus adéquat pour faciliter la mise en place de politiques permettant l'accès des populations aux infrastructures d'eau potable. Cette étude basée essentiellement sur l'utilisation des SIG constitue une contribution importante dans l'étude de l'approvisionnement en eau dans les Communes du Sud du Bénin en particulier dans la Commune de Kétou. Les SIG ont permis de faire d'abord un état des lieux de la répartition des ouvrages dans la Commune. Les types d'ouvrages et leur état de fonctionnement ont été identifiés par le biais des cartes d'inventaires. Puis l'accessibilité aux points d'eau dans chaque arrondissement et la population desservie par les ouvrages a été analysée. Ces résultats ont permis de démontrer l'utilité des SIG pour la gestion des ouvrages d'approvisionnement en eau potable. Cependant, ces avantages du SIG ne doivent pas faire perdre de vue les multiples difficultés liées à la qualité, au nombre souvent insuffisant des données, à leur interpolation et la répartition spatio-temporelle de ces données pour la réalisation d'une bonne étude. Aussi, l'estimation de la population future et du nombre de points d'eau nécessaire à l'horizon 2030 dans des études futures montrera les besoins en eau potable en 2030 .

\section{CONFLIT D'INTERETS}

Les auteurs déclarent qu'ils n'ont aucun conflit d'intérêts pour ce manuscrit.

\section{CONTRIBUTIONS DES AUTEURS}

Ce travail a été initié et réalisé par RANL en collaboration avec les auteurs cidessus cités qui ont supervisé le travail et apporté leurs corrections.

\section{REMERCIEMENTS}

Les auteurs remercient l'Académie de Recherche et d'Enseignement Supérieur (ARES) pour avoir financé le stage en Belgique et ces travaux. Ils remercient tous les membres $\mathrm{du}$ Laboratoire d'Analyse Géospatiale (ANAGEO) pour la formation et le soutien durant toute la période de stage au sein de leur laboratoire.

\section{RÉFÉRENCES}

Abdoulaye A-R. 2017. Utilisation des Systèmes d'Informations Géographiques (SIG) pour une gestion optimale des ressources en eau en Afrique Occidentale: Cas de la commune de Nikki en République du Bénin. International Journal of Innovation and Applied Studies, 19(3): 526-541. http://www.ijias.issr-journals.org/.

Adjovi NRA, Agboton A-AG, Quenum F, Miassi YES, Dossa FK, Adedemi O. 2018. Variation climatique et production vivrière au Sud-Bénin: cas de la 
commune de Bohicon. Afrique Science, 15(2) : 32-43.

Assouma ID, Tohozin CAB, Agbo BF. 2013. Système d'Information Géographique et qualité physico-chimique de l'eau de boisson à Kandi, Bénin. Int. J. Biol. Chem. Sci., 7(5): 2165-2177. DOI: 10.4314/ijbcs.v7i5.32.

Croix Rouge Suisse. 2015. L'eau potable, condition à la santé : la CRS se mobilise en faveur de l'accès à l'eau et de l'hygiène. CRS, $16 \mathrm{p}$.

Direction Générale de l'Eau. 2007. Adductions d'eau villageoises : guide à l'usage des Communes ». Rapport d'étude, DG-Eau, Cotonou, Bénin, 67p.

Direction Générale de l'Eau. 2010. Guide de programmation communale des ouvrages d'approvisionnement en eau potable en milieu rural et semi urbain. Rapport d'étude, DG-Eau, Cotonou, Bénin, 54 pages.

Direction Générale de l'Eau. 2018. Situation des points d'eau au Benin au 30 Décembre 2017. Rapport d'étude, DGEau, Cotonou, Bénin. eaubenin.bj/site/index.php.

El Morjani Z. 2002. Conception d'un système d'information à référence spatiale pour la gestion environnementale : application à la sélection de sites potentiels de stockage de déchets ménagers et industriels en région semi-aride (Souss, Maroc). Thèse de Doctorat, Université de Genève, Terre et Environnement, Suisse, $300 \mathrm{p}$.

Gnazou MDT, Assogba K, Sabi BE, Bawa LM. 2015. Qualité physico-chimique et bactériologique des eaux utilisées dans les écoles de la préfecture de Zio (Togo). Int. J. Biol. Chem. Sci., 9(1): 504-516. DOI:

http://dx.doi.org/10.4314/ijbcs.v9i1.43.

Hounguevou SCG, Tohozin CB, Soumah M, Attolou SFB, Toko IM. 2014. SIG et distribution spatiale des infrastructures hydrauliques dans la commune de Zè au Benin. Afrique Science, 10(2) : 213-227. Hounguevou SCG, Tohozin CB, Soumah M, Attolou SFB. 2014. Approche SIG pour une analyse spatiale des infrastructures hydrauliques dans la Commune de Zè, Benin ». J. Appl. Biosciences, 73: 59495958.

Houeze CR. 2017. Application des SIG pour le suivi et la planification des points d'eau potable dans le Sud-Ouest du Benin. Rapport final du projet d'application du stage en système d'information géographique, Université Libre de Bruxelles, Belgique, 44p.

Ibrahim DA. 2018. Gestion des ouvrages d'hydraulique rurales dans la Commune de Guidan-roumji au Niger, région de Maradi. Rapport final du projet d'application du stage en Système d'Information Géographique, Université Libre de Bruxelles, Belgique, 28 p.

IGIP. 2017. Plan directeur de développement du sous-secteur de l'approvisionnement en eau potable en milieu rural du Bénin. IGIP, Benin, $62 \mathrm{p}$.

Institut National de la Statistique et de l'Analyse Economique (INSAE). 2016. Effectifs de la population des villages et quartiers de ville du Bénin (RGPH4, 2013). INSAE, Benin, 85 p.

Kelome NC, Dovonou L, Lawani RAN. 2014. Problématique de l'eau desservie à la population par les postes d'eau autonomes privés dans la Commune de Ketou. Journal de la Recherche Scientifique de l'Université de Lomé, 16(1) : 15-23.

Kontinental Water Africa. 2018. L'eau potable au Bénin. KWA, Benin. http://www.kwaterafrica.com/2019/02/12/eau-potablebenin/, consulté le 19 Août 2019.

Konan-Waidhet B, Dibi B, Kouadio KE, Savane I, Lazar G. 2013. Apport des techniques géospatiales pour l'analyse de la productivité des aquifères fissurés de 
la région de Dioulatiedougou (NordOuest de la Côte d'Ivoire). Int. J. Biol. Chem. Sci., 7(6): 2611-2625. DOI: http://dx.doi.org/10.4314/ijbcs.v7i6.3.

Laure M. 2018. Un tiers de la population africaine privée d'eau potable : quelles solutions structurelles ? Afrique solutions, Benin. https://www.notreplanete.info/actualites/1846-eau-potableAfrique-solutions, consulté le 19 août 2019.

Michozounnou MMP, Nassi KM, Koumassi DH, Agbossou KE. 2015. Offre du service public d'eau potable à l'épreuve de la poussée démographique dans la Commune d'Adjohoun au Bénin. Afrique Science, 11(4) : 127394922.

Nguyen VB. 2014. Conception d'un SIG pour l'appui à la gestion intégrée des ressources en eau à l'échelle du bassin hydrologique. Thèse de doctorat en Hydrologie, hydrochimie, sol, environnement, Université Toulouse III Paul Sabatier, 256p.

Sokegbe OY, Djeri B, Kogno E, KangniDossou M, Mensah RT, Soncy K,
Ameyapoh Y. 2017. Les risques sanitaires liés aux sources d'eau de boisson dans le district $\mathrm{n}^{\circ} 2$ de Lomécommune : cas du quartier d'Adakpamé. Int. J. Biol. Chem. Sci., 11(5): 23412351.

DOI: http://dx.doi.org/10.4314/ijbcs.v11i5.31 Takem Mbi B. 2017. L'utilité des SIG et l'analyse multicritère pour la planification des infrastructures sanitaires dans le département de Mayo Danay, Région de l'Extrême-Nord, Cameroun. Rapport de stage en SIG libres, Université libre de Bruxelles, Belgique, $25 \mathrm{p}$.

Yemelong TN. 2017. Contribution des Systèmes d'Information Géographique « SIG » au développement des Communes rurales du département des Bamboutos (Ouest-Cameroun) dans un contexte de décentralisation ». Rapport de stage en SIG libres, Université libre de Bruxelles, Belgique, 46p. 